\title{
Proton magnetic resonance spectroscopy pattern of progressive multifocal leukoencephalopathy in AIDS
}

\begin{abstract}
Alex Iranzo, Angel Moreno, Jesus Pujol, Joan Martí-Fàbregas, Pere Domingo, Joan Molet,
\end{abstract} Josep Ris, Josep Cadafalch

\begin{abstract}
The objective was to determine whether the use of intermediate echo times (135 $\mathrm{ms}$ ) in proton magnetic resonance spectroscopy $\left({ }^{1} \mathrm{H}-\mathrm{MRS}\right)$ detects a homogenous pattern in progressive multifocal leukoencephalopathy (PML) in HIV-1 infected people, and to confirm the results of previous studies.

Six patients infected with HIV-1, with PML established by biopsy, and six healthy age and sex matched volunteers were evaluated to define their spectroscopic pattern. ${ }^{1} \mathrm{H}-\mathrm{MRS}$ spectra performed at $1.5 \mathrm{~T}$ were obtained with the STEAM sequence: TE/TM/TR, $20 \mathrm{~ms} / 13.7$ $\mathrm{ms} / 2000 \mathrm{~ms} ; 2500 \mathrm{~Hz}$, size 2048 points, 256 acquisitions (STEAM-20) and with the PRESS sequence; TE/TR, $135 \mathrm{~ms} / 2000 \mathrm{~ms}$; $2500 \mathrm{~Hz}$, size 2048 points, 256 acquisitions (PRESS-135). A single voxel was placed on the lesions and on the parieto-occipital white matter of controls. The peaks of N-acetylaspartate (NAA), choline (Cho), myoinositol (mI), lactate, and lipids were considered, and the results were expressed using creatine as reference.

Spectra of PML lesions were characterised by significantly reduced NAA, lactate presence, and by significantly increased Cho and lipids compared with control group values.

These results indicate that ${ }^{1} \mathrm{H}-\mathrm{MRS}$ detects a homogenous pattern in PML lesions. Recent studies, together with this, suggest that ${ }^{1} \mathrm{H}-M R S$ may help in the diagnostic approach to patients with suspected PML lesions associated with AIDS. (F Neurol Neurosurg Psychiatry 1999;66:520-523)
\end{abstract}

Keywords: proton magnetic resonance; AIDS; progressive multifocal leukoencephalopathy

Correspondence to: Dr Alex Iranzo, Servei de Neurologia, Hospital Clinic, C/Villarroel 170, Barcelona 08036, Spain. Telephone 00343 2275413; fax 00343 2275454

Received 3 March 1998 and in revised form

11 September 1998

Accepted 22 September

1998
Neurological and radiological findings are non-specific. ${ }^{34}$ Serological studies are not helpful in the diagnosis of $\mathrm{PML}^{1}$ and JCV polymerase chain reaction in CSF may be limited by the use of different methods and scarce clinical experience. ${ }^{5}$ Although a definitive diagnosis can only be established by brain biopsy, this method is non-diagnostic in $4 \%$ to $36 \%$ of patients with AIDS. ${ }^{6}$ Thus a non-invasive diagnostic method would obviate the need for brain biopsy in PML suspected lesions provided it is sufficiently specific and sensitive.

Proton magnetic resonance spectroscopy $\left({ }^{1} \mathrm{H}-\mathrm{MRS}\right)$ obtains non-invasive "metabolic biopsies" of living tissues, and correlates metabolic alterations with pathology. ${ }^{7}$ The aim of the present study was to evaluate the homogeneity of the spectroscopic pattern of PML lesions established at biopsy at short $(20 \mathrm{~ms})$ and intermediate $(135 \mathrm{~ms})$ echo times to establish whether this method provides a sensitive pattern for its future diagnostic use. In comparison with previous studies, ${ }^{8-10}$ we used slightly different acquisition parameters to maximise detection of cerebral metabolites.

\section{Methods}

PATIENTS

Studies were performed in six patients with HIV-1 with pathologically established PML lesions and in six healthy age matched controls. HIV-1 infection was documented by enzyme linked immunosorbent assay (ELISA) and confirmed by western blot analysis. ${ }^{11}$ The diagnosis of AIDS was based on the clinical criteria established by the Centers for Disease Control. ${ }^{12}$ In all cases, neurological examination, laboratory data, MRI with and without contrast, and ${ }^{1} \mathrm{H}-\mathrm{MRS}$ were recorded. The indication for biopsy was determined according to radiologically detectable lesions with no regression tendency in patients having antitoxoplasmic therapy, or white matter focal lesions. All six patients included in this study gave their written consent for brain biopsy, MRI, and ${ }^{1} \mathrm{H}-\mathrm{MRS}$.

Control subjects were healthy volunteers matched by age and sex selected from the hospital staff. 


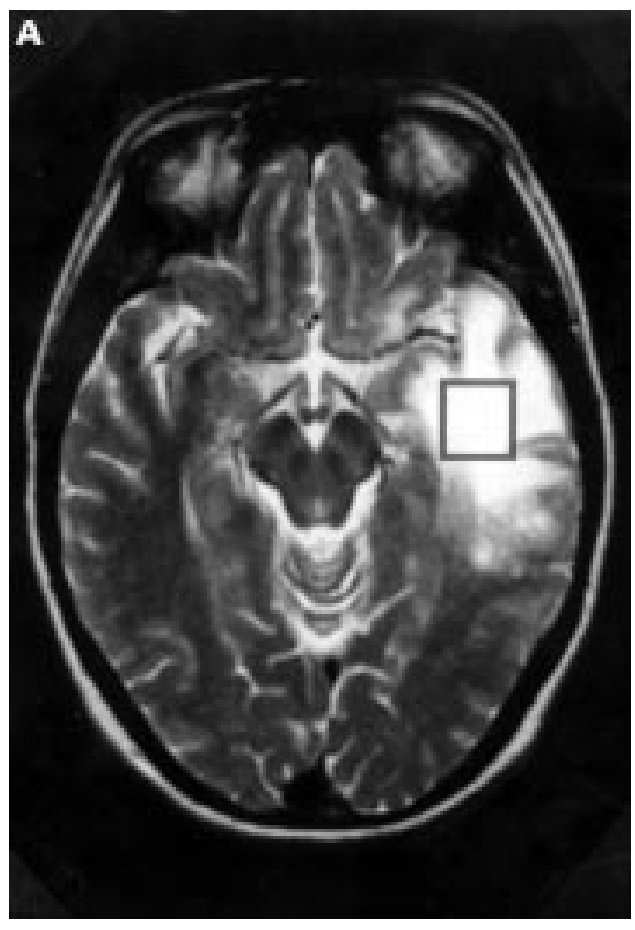

B

PRESS-135

STEAM-20
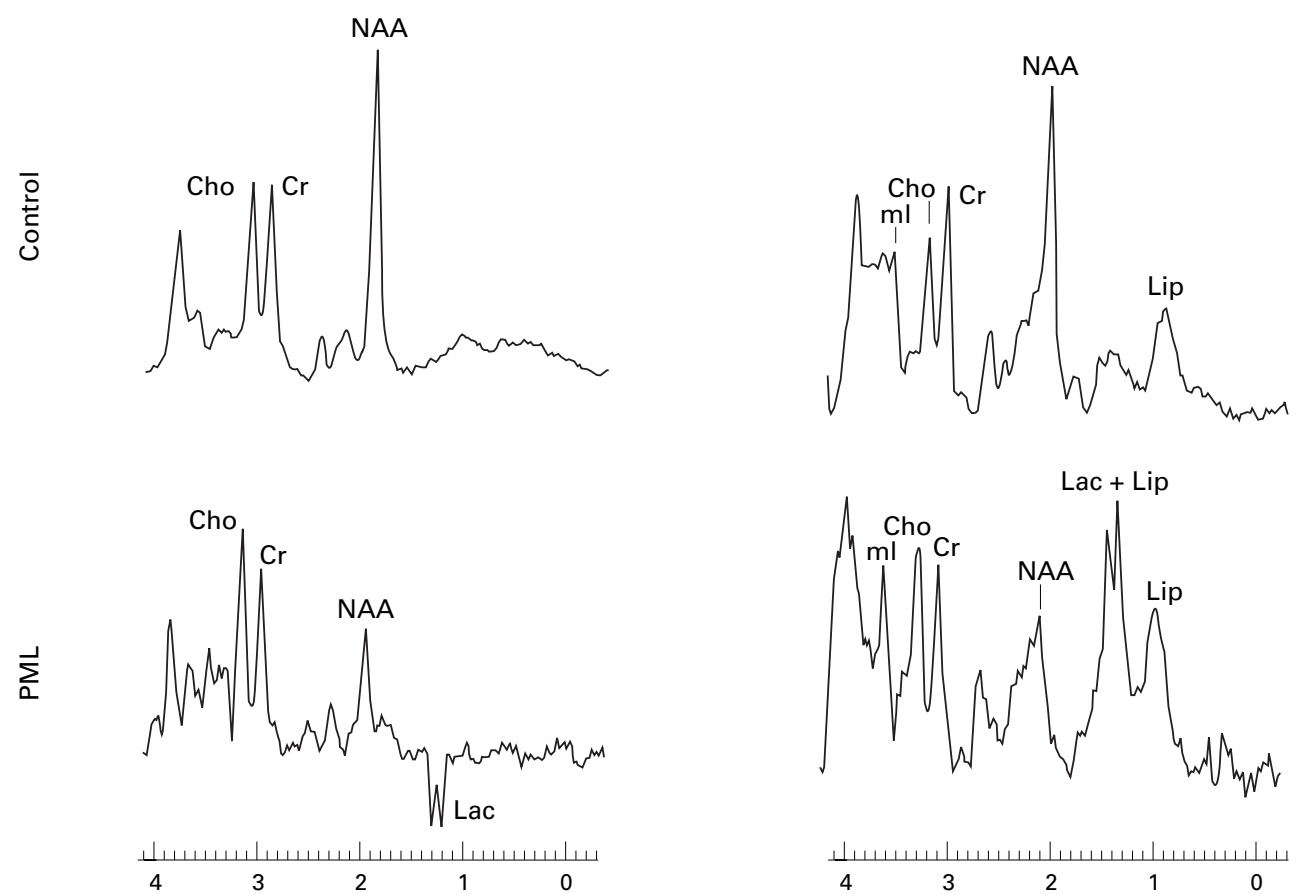

ppm

(A) T2 weighted axial MRI shows a characteristic temporal PML lesion with the voxel placement centred in the lesion. (B) In vivo localised ${ }^{1} \mathrm{H}$-MRS spectra from white matter control subjects (Control) and PML lesions in HIV-1 infected patients (PML). In comparison with normal white matter, note the strong increase of lipids (Lip) and lactate (Lac), the mild increase of choline (Cho) and myo-inositol (mI), and the evident decrease of N-acetylaspartate (NAA) in PML lesions.

MR STUDIES

Both, MRI and ${ }^{1} \mathrm{H}-\mathrm{MRS}$, were performed before brain biopsy. Localised ${ }^{1} \mathrm{H}-\mathrm{MRS}$ was conducted immediately after MRI. MRI studies were performed using a Signa System 1.5 T (General Electric Medical Systems, Milwau- kee, WI, USA) and a quadrature head coil as previously described. ${ }^{13}$ The MRI study was based on coronal, sagittal, and axial projections using spin echo sequences.

${ }^{1} \mathrm{H}-\mathrm{MRS}$ consisted of a "stimulated echo acquisition method" (STEAM) pulse sequence 
(TR/TE 1,600/20 ms; TM $13.7 \mathrm{~ms} ; 256$ acquisitions; 2048 data points; $2500 \mathrm{~Hz}$ spectral width) and a "point resolved spectroscopy" (PRESS) sequence (TR/TE 1600, 135 ms, 256 acquisitions, 2048 data points, and $2500 \mathrm{~Hz}$ spectral width). Both sequences were preceded by three chemical shift water suppression pulses ( $50 \mathrm{~Hz}$ bandwidth). These pulse sequences were obtained with the single voxel proton brain examination (PROBE/SV) system, which automatically optimises magnetic field homogeneity over the selected voxel and adjusts the water suppression pulses. A single voxel was placed on the lesions, adjusting the voxel size to the size and shape of the lesions (figure $\mathrm{A}$ ), and on parieto-occipital white matter in controls $\left(8 \mathrm{~cm}^{3}\right)$.

Data processing was performed on a workstation (SPARCstation 20; Sun Microsystems, Mountain View, CA, USA). Peak areas were estimated with an analysis of free induction delays as described by Saunders et al. ${ }^{14}$ Thus the amplitudes of the time domain signals, which correspond to the area under each resonance in the frequency domain were measured. The main steps of this analysis involve removal of residual water using the Hankel Lanczos singular value decomposition (HLSVD) algorithm ${ }^{15}$ and non-linear least squares fitting using the variable projection (VARPRO) method. ${ }^{16}$

Results were expressed as ratios using creatine as reference. Measurements were performed at the following resonances:

$\mathrm{N}$-acetylaspartate (NAA) (2.01 ppm), creatine $(\mathrm{Cr})$ (3.04 ppm), choline (Cho) (3.22 ppm), myoinositol (mI) (3.55 ppm), lactate (1.33 ppm), and lipids (0.90 ppm).

Statistical analysis was performed with Student's $t$ test.

PATHOLOGICAL DIAGNOSIS

Diagnosis of PML was established by brain biopsy in all patients as previously described. ${ }^{17}{ }^{18}$ The lesion used for pathological evaluation was the same as that on which ${ }^{1} \mathrm{H}-\mathrm{MRS}$ had previously been performed.Time between ${ }^{1} \mathrm{H}$-MRS examination and pathological analysis ranged from 4 to 8 days.

\section{Results}

CLINICAL FINDINGS

Two men and four women, with a mean age of 38.6 (SD 7.0) years (range 30-51 years) were studied. Risk factors for HIV-1 infection were intravenous drug misuse (four patients) and heterosexual transmission (two patients). In two patients, PML was the AIDS defining illness. The mean CD4 count was 40.5 (SD 24.1) $\mathrm{mm}^{3}$ (range $8-76 \mathrm{~mm}^{3}$ ). Two patients presented with Balint's syndrome, one with cortical blindness, and three with both aphasia and right hemiparesis.

\section{MR FINDINGS}

Brain MRI showed a single lesion in two patients and multiple lesions in four patients, all located in the subcortical white matter. Lesions consisted of areas of hypointensity on
${ }^{1} H$-MRS findings

\begin{tabular}{llll}
\hline & $\begin{array}{l}\text { PML } \\
\text { (mean (SD)) }\end{array}$ & $\begin{array}{l}\text { Control } \\
\text { subjects } \\
\text { (mean (SD)) }\end{array}$ & $p$ Value \\
\hline STEAM-20: & & & \\
$\quad$ Lipids (0.9)/Cr & $1.61(0.42)$ & $0.23(0.40)$ & 0.01 \\
Lactate+lipids / Cr & $1.31(0.38)$ & 0 & $\left({ }^{\star}\right)$ \\
NAA / Cr & $0.78(0.21)$ & $1.37(0.10)$ & 0.01 \\
Cho / Cr & $1.23(0.19)$ & $0.92(0.11)$ & 0.01 \\
mI / Cr & $0.76(0.12)$ & $0.65(0.07)$ & $\mathrm{NS}$ \\
PRESS-135: & & & \\
Lactate/Cr & $0.39(0.17)$ & 0 & $\left({ }^{\star}\right)$ \\
NAA/Cr & $0.77(0.09)$ & $2.01(0.22)$ & 0.01 \\
Cho/Cr & $1.53(0.26)$ & $1.17(0.22)$ & 0.04 \\
\hline
\end{tabular}

Metabolite ratios calculated from the STEAM-20 and PRESS135 spectra of parieto-occipital white matter of control subjects and patients with PML lesions. Values are expressed as mean (SD).

${ }^{\star}$ ) Differences were qualitative.

T1 and hyperintensity on T2 weighted images, without mass effect or gadolinium enhancement (figure A).

Spectroscopic examinations were well tolerated by all patients and volunteers, and good quality data were recorded in all studies. Figure B shows representative spectra from PML lesions and normal white matter. PRESS sequence allowed an unequivocal assignation of lactate due to its characteristic phase modulation and removal from lipid resonances at the echo time used. The short echo time used in the STEAM sequence allowed the observation of lipid resonances. By comparison with normal white matter, the main differences found in PML lesion spectra (table) were the presence of lactate in all cases at $1.33 \mathrm{ppm}$, which in STEAM-20 also involves lipids, a significant decrease in the $\mathrm{NAA} / \mathrm{Cr}$ ratio, and a significant increase in $\mathrm{Cho} / \mathrm{Cr}$, and lipids (STEAM-20 spectra)/Cr ratio values.

PATHOLOGICAL FINDINGS

In all patients image guided biopsies were diagnostic showing areas of demyelination with axonal sparing, inclusions within swollen oligodendrocytes, astrocytosis, and enlarged multinucleated astrocytes with bizarre and mitotic nuclei. In situ hybridisation (ISH) for JCV showed DNA in the nuclei of oligodendrocytes and astrocytes in all the patients.

\section{Discussion}

In all PML lesions results showed a homogenous spectroscopic pattern which was characterised by a decrease of NAA/Cr ratio and an increase of lac/Cr, Cho/Cr, and lipids/Cr ratios compared with healthy control subjects. This pattern, in agreement with the results of Chang et $a l,{ }^{9} 10$ could be interpreted as a decrease of NAA, lactate presence, and an increase in Cho and lipids. However, as creatine concentration may be decreased in PML, ${ }^{910}$ the other metabolites would seem to be higher than the actual concentration. The findings of Chang et $a l^{9^{10}}$ and ours suggest that ${ }^{1} \mathrm{H}-\mathrm{MRS}$ may support the diagnostic approach in patients with HIV with suspected PML.

We used the $135 \mathrm{~ms}$ echo time in the PRESS sequence. This intermediate echo time has the advantage of offering better signal to noise ratio 
in resonances of normal brain metabolites, and a higher visibility and unequivocal assignation of lactate due to characteristic phase modulation. Precisely, the use of long echo times produces a strong reduction of resonance intensity that at low concentrations of the metabolites responsible may be indistinguishable from the spectral noise level.

In our study, ${ }^{1} \mathrm{H}-\mathrm{MRS}$ results were obtained from pathologically established PML lesions. Because ${ }^{1} \mathrm{H}$-MRS detects abnormalities in asymptomatic patients infected with $\mathrm{HIV},{ }^{19}$ we compared spectra of PML lesions with those in normal brain parenchyma of healthy subjects and not with their contralateral hemisphere.

Our findings are consistent with neuropathological data. As NAA is a neuronal marker, ${ }^{7}$ its decrease in PML lesions could be the result of the axonal loss shown by biopsy. Lactate was detected in all PML lesions, both in short and long echo time. The lactate presence is related to cellular hypoxia and decreased mitochondrial ATP synthesis. ${ }^{7}$ In PML, the origin of the lactate peak may arise from the presence of large numbers of microglial phagocytes and necrotic oligodendrocytes as described in acute

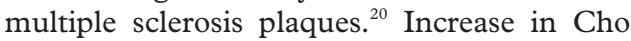
resonance (glycerophosphocholine, phosphocholine, choline) and lipids may reflect the accumulation of myelin breakdown products and rapid cell membrane synthesis. ${ }^{7}$ Both processes, myelin breakdown and enlarged bizarre astrocytes (which were seen undergoing mitosis and malignant in aspect), have been found in all our neuropathological analyses of PML biopsies. PML lesions showed raised $\mathrm{mI}$, though not significantly, compared with normal controls. As $\mathrm{mI}$ is present within neuroglial cells, ${ }^{21}$ its increase may reflect the astrocytic proliferation in all our six patients.

In summary, our results reflect the neuropathological processes and show a homogeneous and uniform spectroscopic pattern in PML lesions established by biopsy. Even though ours is a small series, these data suggest that ${ }^{1} \mathrm{H}-\mathrm{MRS}$ may help as a non-invasive examination to differentiate between PML and other focal brain lesions in HIV infected patients. However, further work will be necessary to evaluate the discriminative ability of our indices in the study of HIV related intracerebral lesions.
This study was supported by a grant from Sant Pau-Citran.

1 Berger JR, Gallo BV, Concha M. Progressive multifocal leukoencephalopathy. In: Berger JR, Levy RM, eds. AIDS and the nervous system. Philadelphia: Lippincott-Raven, 1997:569-94.

2 Berger JR, Kasovitz B, Post MJD, et al. Progressive multifocal leukoencephalopathy associated with human immunodeficiency virus. A review of the literature with a report of sixteen cases. Ann Intern Med 1987;107:78-87.

3 Mark AS, Atlas SW. Progressive multifocal leukoencephaMark AS, Atlas SW. Progressive multifocal leukoencephalopathy in patients with AID
Radiology 1989;173:517-20.

4 Wheeler AL, Truwit CL, Kleinschmidt-DeMasters BK, et al. Progressive multifocal leukoencephalopathy: contrast enhancement on CT scans and MR images. AfR Am $\mathcal{F}$ Roentgenol 1993;161:1049-51.

5 Cinque P, Scarpellini P, Vago L, et al. Diagnosis of central nervous system complications in HIV infected patients: cerebrospinal fluid analysis by the polymerase chain reaction. AIDS 1997;11:1-17

6 Holloway RG, Mushlin AI. Intracranial mass lesions in acquired immunodeficiency syndrome: using decision analysis to determine the effectiveness of stereotactic biopsy. Neurology 1996;46:1010-15.

7 Ross BD, Michaelis T. Clinical applications of magnetic resonance spectroscopy. Magn Reson Q 1994;10:191-247.

8 Confort-Gouny S, Vion-Dury J, Nivoli $\mathrm{F}$, et al. A multiparametric data analysis showing the potential of localized prometric data analysis showing the potential of localized pro-
ton MR spectroscopy of the brain in the metabolic characterization of neurological diseases. $\mathcal{f}$ Neurol Sci 1993;118: terization

9 Chang L, Miller BL, McBride D, et al. Brain lesions in patients with AIDS: H-1 MR Spectroscopy. Radiology 1995; 197:525-31

10 Chang L, Ernst T, Tornatore C, et al. Metabolite abnormalities in progressive multifocal leukoencephalopathy by proton magnetic resonance spectroscopy. Neurology 1997;48:836-45.

11 Centers for Disease Control. Update: serologic testing for HIV-1 antibody. MMWR 1990;39:380-3.

12 Centers for Disease Control and Prevention. 1993 Revised classification system for HIV infection and expanded surveillance case definition of acquired immunodeficiency syndrome among adolescents and adults. JAMA 1993;269: 729-30.

13 Pujol J, Kulisevsky J, Moreno A, et al. Neurospectroscopic alterations and globus pallidus hyperintensity as related alterations and globus pallidus hyperintensity as related
magnetic resonance markers of reversible hepatic encephamagnetic resonance markers of reversibl
lopathy. Neurology 1996;47:1526-30.

14 Saunders DE, Howe FA, van den Boogaart A, et al. Continuing ischemic damage after acute middle cerebral artery infarction in humans demonstrated by short-echo proton spectroscopy. Stroke 1995;26:1007-13.

15 Pijnappel WWF, van den Boogaart A, de Beer R, et al. SVDbased quantification of magnetic resonance signals. $\mathcal{f}$ Magn Reson 1992;97:122-34.

16 van der Veen JWC, de Beer R, Luyten PR, et al. Accurate quantification of in vivo P-31 NMR signals using the variable projection method and prior knowledge. Magn Reson Med 1988;6:92-8.

17 Hunter EE. Practical electron microscopy: a beginner's illustrated guide. New York: Praeger, 1984.

18 Askamit AJ, Mourrain P, Sever JL, et al. Progressive multifocal leukoencephalopathy: investigation of three cases using in situ hybridation with JC virus biotinylated DNA probe. Ann Neurol 1985;18:490-6.

19 Wilkinson ID, Miller RF, Miszkiel KA, et al. Cerebral proton magnetic resonance spectroscopy in assymptomatic HIV infection. AIDS 1997;11:289-95.

20 Miller DH, Austin SJ, Connelly, et al. Proton magnetic resonance spectroscopy of an acute and chronic lesion in multiple sclerosis. Lancet 1991;337:58-9.

21 Brand A, Richter-Landsberg C, Leibrift D. Multinuclear NMR studies on the energy metabolism of glial and neuronal cells. Dev Neurosci 1993;15:289-98. 